\title{
Topological susceptibility of two-dimensional $U(N)$ gauge theories
}

\author{
Claudio Bonati ${ }^{*}$ and Paolo Rossi ${ }^{\dagger}$ \\ Dipartimento di Fisica, Università di Pisa and INFN, \\ Sezione di Pisa, Largo Pontecorvo 3, 56127 Pisa, Italy
}

(Received 1 February 2019; published 18 March 2019)

\begin{abstract}
In this paper, we study the topological susceptibility of two-dimensional $U(N)$ gauge theories. We provide explicit expressions for the partition function and the topological susceptibility at finite lattice spacing and finite volume. We then examine the particularly simple case of the Abelian $U(1)$ theory, the continuum limit, and the infinite volume limit, and we finally discuss the large $N$ limit of our results.
\end{abstract}

DOI: 10.1103/PhysRevD.99.054503

\section{INTRODUCTION}

The study of $\theta$ dependence of QCD by means of lattice simulations has been the subject of several recent studies, mainly triggered by the possible implications for axion physics [1-9]. It is, however, well known that Monte Carlo algorithms typically used in numerical simulations suffer from a severe critical slowing down as the continuum limit is approached, with autocorrelation times of topological observables that grow about exponentially in the inverse of the lattice spacing [10-13]. This has led to the development of new algorithms, specifically devised to improve the sampling of topologically nontrivial configuration [7,8,14-23].

From a general point of view, it is very useful to have the possibility of performing quantitative checks of the Monte Carlo results against exact ones. This is obviously not possible in the general case; however, simplified (toy) models that are analytically soluble but still complicated enough to be used as nontrivial test beds sometimes exist. In statistical physics, the two-dimensional Ising model is probably the most popular choice [24], while in field theory, two-dimensional lattice gauge theories are the natural playground for tests of numerical simulations; on one side, they are computationally much cheaper than their four-dimensional counterparts, and on the other side, it is possible to determine many exact results that may constitute precise benchmarks for numerical results and extrapolations.

The present paper is devoted to the extension of known analytic results concerning two-dimensional $U(N)$ lattice

\footnotetext{
claudio.bonati@df.unipi.it

paolo.rossi@unipi.it
}

Published by the American Physical Society under the terms of the Creative Commons Attribution 4.0 International license. Further distribution of this work must maintain attribution to the author(s) and the published article's title, journal citation, and DOI. Funded by SCOAP ${ }^{3}$. gauge theories in the absence of a $\theta$ term to the case in which such a term is present, and more specifically to the evaluation of the topological susceptibility, for finite volumes $V$ and for generic values of the coupling $\beta$. Finite volume results at fixed coupling may be especially useful because they allow direct comparison with simulations without the need for extrapolating to infinite volume and to the continuum limit.

The paper is organized as follows. Section II is devoted to a summary of known results, with special emphasis on finite lattices with spherical and toroidal geometries. In Sec. III we fix our notation, trying to make correspondence with previous literature as far as possible. We give our definitions for the density of topological charge and for the topological susceptibility in $U(N)$ gauge theories, exploiting the existence of the $U(1)$ subgroup. We present our general formulas for the partition function in the presence of a $\theta$ term and for the topological susceptibility; for generic values of $N, \beta$, and $V$; and for any genus $g$ of the lattice manifold, showing explicitly that the periodicity of the partition function for $2 \pi$ shifts of the $\theta$ parameter is preserved. In Sec. IV, we focus on the case $N=1$ where many closed-form expressions can be explicitly found for generic values of $V$ and can be compared with partial results already available in the literature. Strong evidence of precocious scaling by using a renormalized coupling is also exhibited. In Sec. V, we analyze the (finite volume) continuum limit of the model $\beta \rightarrow \infty$ in the presence of a $\theta$ term. In Sec. VI, the infinite volume limit of the topological susceptibility is discussed in detail. Section VII is devoted to the study of the large $N$ limit in the infinite and finite volume cases (with further evidence of precocious scaling) and to numerical checks of our large $N$ results.

\section{SUMMARY OF KNOWN RESULTS}

The finite volume lattice version of $U(N)$ gauge theories most widely studied in the literature is defined by the following partition function [25], 


$$
\begin{gathered}
Z(N, \beta, P)=\int e^{-S(N, \beta, P)} \prod_{l=1}^{L} \mathrm{~d} U_{l}, \\
S(N, \beta, P)=-N \beta \sum_{p=1}^{P} \operatorname{Tr}\left(U_{p}+U_{p}^{\dagger}\right),
\end{gathered}
$$

where unitary $N \times N$ matrices $U_{l}$ are attached to the $L$ links of the lattice and $U_{p}=\prod U_{l}$ are the ordered products of the link matrices along any lattice plaquette. $\beta$ is the lattice 't Hooft coupling, the relationship with the standard (dimensionful) coupling ${ }^{1}$ of which is $N \beta=1 /\left(g^{2} a^{2}\right)$, where $a$ is the lattice spacing and the volume is given by $V=P a^{2}$. The sum in Eq. (2) runs over all $P$ plaquettes, while the integration $\mathrm{d} U_{l}$ involves all link variables and is performed by using the Haar measure for the $U(N)$ group. Due to its crucial role, we recall that, when the integrand involves only functions of the eigenvalues $\phi_{i}$ of the integration variable, the $U(N)$ Haar measure reduces to (see, e.g., Ref. [26])

$$
\mathrm{d} \mu(\phi)=\Delta(\phi) \bar{\Delta}(\phi) \prod_{i=1}^{N} \frac{\mathrm{d} \phi_{i}}{2 \pi}
$$

where

$$
\Delta(\phi)=\frac{1}{\sqrt{N !}} \epsilon_{j_{1} \cdots j_{N}} e^{i \phi_{1}\left(N-j_{1}\right)} \cdots e^{i \phi_{N}\left(N-j_{N}\right)}
$$

The peculiarity of two-dimensional models consists in the possibility of performing a change of integration variables (exploiting the invariance of the Haar measure) in such a way that most nontrivial integrations involve directly the plaquette matrices. That this is a feasible strategy can be understood, for a two-dimensional compact orientable manifold without boundary, by using the Euler characteristic $2-2 g=S-L+P$, where $S$ is the number of sites (vertices) of the lattice and $g$ is the genus of the lattice manifold. The maximal number of links that can be gauged away (maximal tree) is simply $S-1$, and therefore the number of nontrivial integration variables $I$ is $I=L-S+1=P-1+2 g$.

Two cases particularly useful for applications are the manifolds with the topology of the sphere $(g=0)$ and the manifolds with the topology of the torus $(g=1)$. For the case $g=0$, we have $I=P-1$, implying that one of the plaquette variables may be expressed as a function (actually the product) of all other matrices; in this case, one can easily prove the equivalence of these models to the chiral chains of length $P$ (see also later in this section), in order to use the results available for these systems $[27,28]$. For

\footnotetext{
${ }^{1}$ In the following, we will denote by $g$ also the genus of the manifold on which the theory is defined; the meaning of $g$ should, however, be clear from the context.
}

$g=1$ (the manifolds typically adopted in simulations), we get $I=P+1$, and the independent variables may be chosen to be $P-1$ plaquettes and two other degrees of freedom ("torons"). Integration over the torons may be explicitly carried out [29], and the result leads again to the possibility of expressing the last plaquette as the product of all other variables. This procedure can be generalized without difficulties also to the case of generic topology.

Without belaboring the details, we only quote the final result, due to Rusakov [30] (see also Ref. [29] for the case of the torus): the $\theta=0$ partition function $Z^{(g)}(N, \beta, P)$ corresponding to a compact orientable lattice manifold of genus $g$ without boundary is

$$
Z^{(g)}(N, \beta, P)=\sum_{r} d_{r}^{2-2 g}\left[\frac{\tilde{\beta}_{r}(N, \beta)}{d_{r}}\right]^{P},
$$

where $P>1$, the sum runs over all representations $r$ of $U(N), d_{r}$ is the dimension of the representation, and [26]

$$
\tilde{\beta}_{r}(N, \beta)=\int \chi_{r}(U) e^{N \beta\left(\operatorname{Tr} U+\operatorname{Tr} U^{\dagger}\right)} \mathrm{d} U,
$$

with $\chi_{r}(U)$ the character of $r$. If the manifold is nonorientable, the partition function is always equal to 1 ; if fixed boundaries are present, the result depends on the holonomies associated to the boundaries [30]. When the boundary holonomies are fixed to be trivial, one obtains again Eq. (5), and this is a possible way to prove the equivalence of the spherical topology with chiral chains. We explicitly note that, when writing expressions like Eq. (5), we must keep in mind that the number of links belonging to each plaquette is not a priori fixed, and for small values of $P$, it must be large enough to ensure the possibility of imposing boundary conditions compatible with the genus $g$ of the lattice manifold. In particular, for $P=2$, the plaquettes must be polygons with at least $4 g$ sides.

It is worth noticing that, due to the invariance properties of the measure, a simple result may be obtained in the case $g=0, P=2$ :

$$
Z^{(0)}(N, \beta, 2)=\tilde{\beta}_{0}(N, 2 \beta) .
$$

We also recall that the continuum partition function in the case of a finite (dimensionless) area $A=V / a^{2}$ can be obtained starting from the heat kernel action, corresponding to the replacement ${ }^{2}$ [26]

$$
\tilde{\beta}_{r}(N, \beta) \rightarrow d_{r} e^{-\frac{C_{r}}{4 N \beta}},
$$

\footnotetext{
${ }^{2}$ There is sometimes confusion in the literature on the numerical factor appearing in the exponent, which depends on the conventions adopted in the action. We checked that Eq. (8) is the correct large $\beta$ limit of Eq. (13).
} 
where $C_{r}$ is the quadratic Casimir in the $r$ representation and the result is

$$
Z^{(g)}(N, \beta, A)=\sum_{r} d_{r}^{2-2 g} e^{-\frac{1}{4 N \beta} C_{r} A} .
$$

The infinite volume limit of Eq. (5) can be easily recovered in different ways. For instance, one may observe that when $P \rightarrow \infty$ it is consistent to choose an axial gauge condition, amounting to setting $U_{l}=1$ for all the links in the "time" direction of the lattice. Factorization of the integrals in Eq. (2) follows trivially, implying a direct relationship with the single plaquette model,

$$
Z(N, \beta, P) \stackrel{P \rightarrow \infty}{\rightarrow} Z(N, \beta, 1)^{P},
$$

where

$$
Z(N, \beta, 1) \equiv \int \mathrm{d} U e^{N \beta\left(\operatorname{Tr} U+\operatorname{Tr} U^{\dagger}\right)}=\tilde{\beta}_{0}(N, \beta)
$$

and the properties of the trivial representation $\left[d_{0}=1\right.$ and $\left.\chi_{0}(U)=1\right]$ have been exploited. It is important to stress that the same result might have been obtained by observing that the quantities $\tilde{\beta}_{r}(N, \beta)$ can be explicitly computed for all values of $N$. Indeed, by recalling the definition of the modified Bessel functions of integer order

$$
I_{n}(2 N \beta)=\frac{1}{2 \pi} \int_{-\pi}^{\pi} e^{2 N \beta \cos \phi \pm i n \phi} \mathrm{d} \phi,
$$

it is possible to obtain the result [26]

$$
\tilde{\beta}_{\left\{l_{j}\right\}}(N, \beta)=\operatorname{det}\left(I_{l_{j}+i-j}(2 N \beta)\right),
$$

where the indices $l_{1} \geq \cdots \geq l_{N}\left(l_{i} \in \mathbb{Z}\right)$ parametrize the $U(N)$ representation; in particular [25],

$$
\tilde{\beta}_{0}(N, \beta)=\operatorname{det}\left(I_{i-j}(2 N \beta)\right) .
$$

Noticing that $I_{n}(x)<I_{0}(x)$ for all $n \neq 0$ and for all finite real values of $x$, it is easy to be convinced that

$$
\frac{\tilde{\beta}_{r}(N, \beta)}{d_{r} \tilde{\beta}_{0}(N, \beta)}<1
$$

for all $r \neq 0$ and for all finite values of $\beta$. This observation implies Eq. (10) and also that the convergence to the infinite volume limit is exponentially fast for large values of $P$.

As we mentioned in the Introduction, many exact results have been obtained in the past with regard to the large $N$ limit of many matrix models. For a general review we refer to Ref. [31], quoting here only some results that will be relevant in the following: the existence of a third order phase transition at $N=\infty$, first identified by Gross and
Witten [32] and Wadia [33], the computation of the first few $1 / N$ corrections of the free energy [34], and the exact expression for the large $N$ eigenvalue distribution of the plaquette variable for the single plaquette model [32] and for the chiral chains with $P=3,4[27,28,35]$. The solution of the external field problem for all $N[27,28,36,37]$ and the expectation value of $\operatorname{det} U_{p}$ for the single plaquette model [38].

\section{TOPOLOGICAL CHARGE AND SUSCEPTIBILITY}

The existence of a topological charge in twodimensional $U(N)$ gauge theories is related to the existence of a $U(1)$ Abelian subgroup, that can be parametrized by a phase $\Phi$, related to the determinant of the $U(N)$ matrix by the relationship

$$
\operatorname{det} U=e^{i \Phi} .
$$

It is easy to be convinced that on a compact orientable lattice manifold without boundaries the following property holds,

$$
\sum_{p=1}^{P} \Phi_{p}=0(\bmod 2 \pi),
$$

where $\Phi_{p}$ is the phase associated with the determinant of each plaquette variable. Hence, a simple definition for the topological charge density $q_{p}$ associated with each plaquette is

$$
q_{p} \equiv-\frac{i}{2 \pi} \ln \operatorname{det} U_{p}=-\frac{i}{2 \pi} \operatorname{Tr} \ln U_{p} ;
$$

the total topological charge is $Q(N, P)=\sum q_{p}$, and, because of the above property of $\sum_{p} \Phi_{p}, Q$ can only take integer values. Note that the second equality in Eq. (18) holds only for an appropriate and $U_{p}$-dependent choice of the branch cuts. If, however, the standard $[-\pi, \pi]$ branch is used (as will always be done in the following), the two expressions for the topological charge are generically different, but nevertheless, the corresponding $\theta$-dependent partition functions are the same.

By definition, the (dimensionless) topological susceptibility $\chi_{t}(N, \beta, V)$ is

$$
\chi_{t}(N, \beta, V)=\frac{a^{2}}{V}\left[\left\langle Q^{2}\right\rangle-\langle Q\rangle^{2}\right],
$$

where the expectation values are to be computed at $\theta=0$. The lattice representation of $\chi_{t}$ follows trivially from the above results, recalling that $V=a^{2} P$, and simple parity arguments imply that $\langle Q\rangle=0$; therefore, in practice, we just have to compute $\left\langle Q^{2}\right\rangle / P$. 
The $\theta$-dependent partition function can be defined as

$$
Z_{\theta}(N, \beta, P) \equiv \int e^{i \theta Q(N, P)} e^{-S(N, \beta, P)} \prod_{l=1}^{L} \mathrm{~d} U_{l},
$$

and in order to compute $Z_{\theta}(N, \beta, P)$, we can repeat and adapt Rusakov's procedure. Let us define the quantities

$$
\tilde{\gamma}_{r}(N, \beta, \theta) \equiv \int \chi_{r}(U) e^{\frac{\theta}{2 \pi} \operatorname{Tr} \ln U+N \beta\left(\operatorname{Tr} U+\operatorname{Tr} U^{\dagger}\right)} \mathrm{d} U,
$$

with the property that

$$
\tilde{\gamma}_{r}(N, \beta, 0)=\tilde{\beta}_{r}(N, \beta) .
$$

By choosing an appropriate gauge condition and performing the residual nontrivial integrations, we then obtain our general result for the $\theta$-dependent partition function:

$$
Z_{\theta}^{(g)}(N, \beta, P)=\sum_{r} d_{r}^{2-2 g}\left[\frac{\tilde{\gamma}_{r}(N, \beta, \theta)}{d_{r}}\right]^{P}
$$

Noticing that $\operatorname{Tr} \ln U=i \sum_{j} \phi_{j}$, where $e^{i \phi_{j}}$ are the eigenvalues of $U$, and defining the functions

$$
\mathcal{I}_{\nu}(x) \equiv \frac{1}{2 \pi} \int_{-\pi}^{\pi} e^{i \nu \phi} e^{x \cos \phi} \mathrm{d} \phi,
$$

which for integer indices reduces to modified Bessel functions [see Eq. (12)], we obtain the closed form expression

$$
\tilde{\gamma}_{\left\{l_{j}\right\}}(N, \beta, \theta)=\operatorname{det}\left(\mathcal{I}_{l_{j}+i-j+\frac{\theta}{2 \pi}}(2 N \beta)\right)
$$

using arguments identical to those needed to prove Eqs. (13) and (14) $[25,26,32]$.

From this expression, it follows that $\theta \rightarrow \theta+2 \pi$ is equivalent to $\left\{l_{j}\right\} \rightarrow\left\{l_{j}^{\prime}\right\}$, where $l_{j}^{\prime}=l_{j}+1$; as a consequence, when performing the summation over all representations, each contribution appearing in $Z_{\theta+2 \pi}^{(g)}(N, \beta, P)$ has an identical counterpart in the expression of $Z_{\theta}^{(g)}(N, \beta, P)$, implying exact $2 \pi$ periodicity in $\theta$ for all values of $g, N, \beta$, and $P$. We consider this to be quite nontrivial evidence for the correct normalization of the topological charge in the two-dimensional $U(N)$ gauge theories.

In order to simplify the notation, it is convenient to introduce the weights

$w_{r}^{(g)}(N, \beta, P)=d_{r}^{2-2 g}\left[\frac{\tilde{\beta}_{r}(N, \beta)}{d_{r}}\right]^{P}\left[Z_{0}(N, \beta, P)\right]^{-1}$,

with the property that $\sum_{r} w_{r}^{(g)}(N, \beta, P)=1$. Starting from the formal expression for the topological susceptibility,

$$
\chi_{t}^{(g)}(N, \beta, P)=-\left.\frac{1}{P} \frac{\partial^{2} \ln Z_{\theta}^{(g)}(N, \beta, P)}{\partial \theta^{2}}\right|_{\theta=0},
$$

it is then possible to represent $\chi_{t}^{(g)}(N, \beta, P)$ in the form

$$
\begin{aligned}
\chi_{t}^{(g)}(N, \beta, P)= & -\sum_{r} w_{r}^{(g)}(N, \beta, P) \frac{\tilde{\gamma}_{r}^{\prime \prime}(N, \beta)}{d_{r} \tilde{\beta}_{r}(N, \beta)} \\
& +-(P-1) \sum_{r} w_{r}^{(g)}(N, \beta, P)\left[\frac{\tilde{\gamma}_{r}^{\prime}(N, \beta)}{d_{r} \tilde{\beta}_{r}(N, \beta)}\right]^{2},
\end{aligned}
$$

where we have defined

$$
\begin{aligned}
\tilde{\gamma}_{r}^{\prime}(N, \beta) & \left.\equiv \frac{\partial \tilde{\gamma}_{r}(N, \beta, \theta)}{\partial \theta}\right|_{\theta=0} \\
& =\int \chi_{r}(U)\left(\frac{1}{2 \pi} \operatorname{Tr} \ln U\right) e^{N \beta\left(\operatorname{Tr} U+\operatorname{Tr} U^{\dagger}\right)} \mathrm{d} U
\end{aligned}
$$

and

$$
\begin{aligned}
\tilde{\gamma}_{r}^{\prime \prime}(N, \beta) & \left.\equiv \frac{\partial^{2} \tilde{\gamma}_{r}(N, \beta, \theta)}{\partial \theta^{2}}\right|_{\theta=0} \\
& =\int \chi_{r}(U)\left(\frac{1}{2 \pi} \operatorname{Tr} \ln U\right)^{2} e^{N \beta\left(\operatorname{Tr} U+\operatorname{Tr} U^{\dagger}\right)} \mathrm{d} U
\end{aligned}
$$

which can be rewritten as sums of determinants involving modified Bessel functions and related functions (see Sec. VI for more details on the simplest case). In the derivation of Eq. (28), we have also exploited the fact that $\langle Q\rangle$ vanishes at $\theta=0$, which is equivalent to

$$
\sum_{r} w_{r}^{(g)}(N, \beta, P) \frac{\tilde{\gamma}_{r}^{\prime}(N, \beta)}{d_{r} \tilde{\beta}_{r}(N, \beta)}=0
$$

The proof of this identity rests on the cancellation of the contributions coming from each representation $r$ (associated to $\left\{l_{j}\right\}$ ) and its conjugate representation $r^{*}$ (associated to $\left.\left\{-l_{N+1-j}\right\}\right)$; indeed,

$$
\begin{aligned}
d_{r^{*}} & =d_{r} ; \quad \tilde{\beta}_{r^{*}}(N, \beta)=\tilde{\beta}_{r}(N, \beta), \\
\tilde{\gamma}_{r^{*}}(N, \beta, \theta) & =\tilde{\gamma}_{r}(N, \beta,-\theta),
\end{aligned}
$$

from which it follows that $\tilde{\gamma}_{r^{*}}^{\prime}(N, \beta)=-\tilde{\gamma}_{r}^{\prime}(N, \beta)$.

By the same arguments applied in the previous section, and observing that $\tilde{\gamma}_{0}^{\prime}(N, \beta)=0$ for obvious symmetry reasons, we may conclude that also the convergence of the topological susceptibility to its infinite volume limit is exponentially fast. An example of the finite volume behavior of the topological susceptibility is shown in Fig. 1 for the $U(2)$ case. 


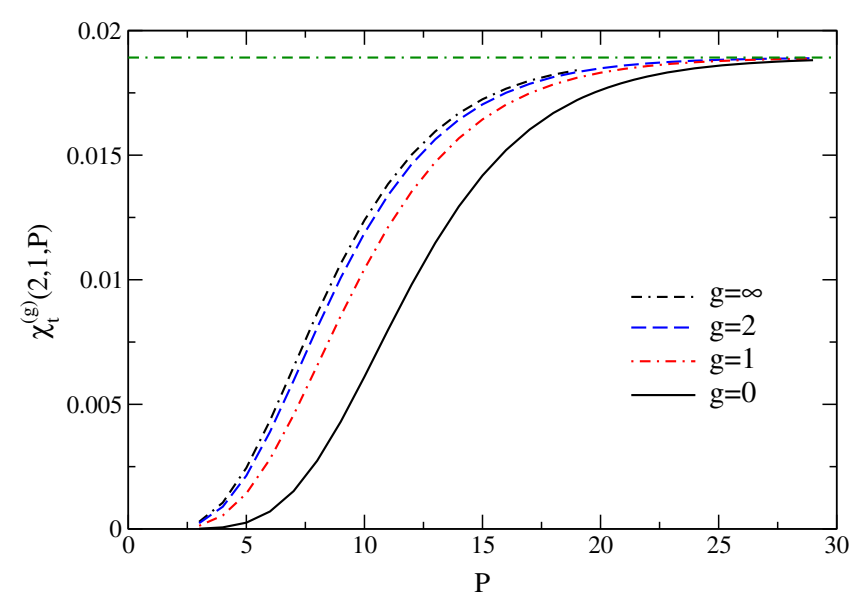

FIG. 1. Behavior of the $U(2)$ topological susceptibility as a function of the dimensionless volume $P$ for the value $\beta=1$ of the coupling. Results are shown for three different topologies, corresponding to $g=0,1$ and 2 ; the horizontal line denotes the asymptotic $P \rightarrow \infty$ value computed by using Eq. (63).

A peculiar property of the case $g=0, P=2$ (the twolink chiral chain) is

$$
\chi_{t}^{(0)}(N, \beta, 2)=0
$$

implied by the trivial relationship $\operatorname{Tr} \ln U+\operatorname{Tr} \ln U^{\dagger}=0$.

It is also quite interesting to study the limit $g \rightarrow \infty$ of the theory. Since $g$ appears in the exponent of $1 / d_{r}$ in the weights $w_{r}^{(g)}$ (see Eq. (26)), all representations with $d_{r}>1$ disappear as $g \rightarrow \infty$, and only the representations labeled with $l_{1}=l_{2} \cdots=l_{N}$ give a finite contribution in this limit. The relevant representations are therefore identified by a single index $l$, running from $-\infty$ to $+\infty$, and the partition function is simply

$$
Z_{\theta}^{(\infty)}(N, \beta, P)=\sum_{l}\left[\tilde{\gamma}_{l}(N, \beta, \theta)\right]^{P}
$$

where the explicit form of the $U(N)$ characters has been used (see Ref. [26]) and

$$
\tilde{\gamma}_{l}(N, \beta, \theta)=\tilde{\beta}_{0}(N, \beta)\left\langle(\operatorname{det} U)^{l+\frac{\theta}{2 \pi}}\right\rangle,
$$

where the average \langle\rangle stands for the average in the single plaquette model at $\theta=0$. The topological susceptibility of the $g=\infty$ theory is shown in Fig. 1 for the $U(2)$ case. Further aspects of the large $g$ behavior will be discussed in Secs. V and VII.

To better understand the form of Eq. (28), it is convenient to further generalize the problem, by introducing plaquettedependent lattice coupling and the $\theta$ angle. It is immediate to verify that the Rusakov result can be generalized to this case and the partition function becomes

$$
Z_{\vec{\theta}}^{(g)}(N, \vec{\beta}, P)=\sum_{r} d_{r}^{2-2 g} \prod_{p=1}^{P} \frac{\tilde{\gamma}_{r}\left(N, \beta_{p}, \theta_{p}\right)}{d_{r}} .
$$

We can now write a formal expression for the two-point correlation function of the topological charge by using

$$
\left\langle q_{i} q_{j}\right\rangle^{(g)}(N, \beta, P)=-\left.\frac{\partial^{2}}{\partial \theta_{i} \partial \theta_{j}} \ln Z_{\vec{\theta}}^{(g)}(N, \vec{\beta}, P)\right|_{\substack{\theta_{p}=0 \\ \beta_{p}=\beta}},
$$

and it is simple to verify that $\left\langle q_{i} q_{j}\right\rangle^{(g)}(N, \beta, P)$ has the form

$$
\begin{aligned}
\left\langle q_{i} q_{j}\right\rangle^{(g)}(N, \beta, P)= & c_{1}^{(g)}(N, \beta, P) \delta_{i j} \\
& +c_{2}^{(g)}(N, \beta, P)\left(1-\delta_{i j}\right),
\end{aligned}
$$

which expresses the fact that in two dimensions the correlator $\left\langle q_{i} q_{j}\right\rangle^{(g)}$ takes just two values. These values are obviously related to the expressions appearing in Eq. (28), that can indeed be rewritten in the form

$\chi_{t}^{(g)}(N, \beta, P)=c_{1}^{(g)}(N, \beta, P)+(P-1) c_{2}^{(g)}(N, \beta, P)$.

This is nothing but the general relation between the susceptibility and the two-point function, written in the case in which $\left\langle q_{i} q_{j}\right\rangle^{(g)}$ assumes only two values. Since $\tilde{\gamma}_{0}^{\prime}(N, \beta)=0$, it is simple to show that $c_{2}$ goes to zero exponentially in $P$ (the dimensionless volume) as the thermodynamic limit is approached; in this limit, the two-point function of the topological charge reduces to a $\delta$ function.

\section{CASE $N=1$}

In the purely Abelian case $N=1$, many simplifications occur, due to the commutativity of the matrices. In particular, there is no dependence on the genus of the manifold, as one can easily see by noticing that all the representations have dimension 1.

The topological charge density is simply $q_{p}=\frac{\phi_{p}}{2 \pi}$ (where $\phi_{p}$ is the Abelian phase of the plaquette), and the character of the $n$th representation of $U_{p}$ is just $e^{i n \phi_{p}}$. As a consequence, one may compute directly the $\theta$-dependent partition function on a finite lattice, obtaining

$$
Z_{\theta}(1, \beta, P)=\sum_{n=-\infty}^{+\infty}\left[\mathcal{I}_{n+\frac{\theta}{2 \pi}}\right]^{P} .
$$

The $U(1)$ weights are simply

$$
w_{n}(1, \beta, P)=\frac{\left[I_{n}(2 \beta)\right]^{P}}{\sum_{n}\left[I_{n}(2 \beta)\right]^{P}} .
$$

The resulting expression for the finite volume topological susceptibility is then 


$$
\begin{aligned}
\chi_{t}(1, \beta, P)= & -\sum_{n} w_{n}(1, \beta, P) \frac{\mathcal{I}_{n}^{\prime \prime}(2 \beta)}{I_{n}(2 \beta)} \\
& +-(P-1) \sum_{n} w_{n}(1, \beta, P)\left[\frac{\mathcal{I}_{n}^{\prime}(2 \beta)}{I_{n}(2 \beta)}\right]^{2},
\end{aligned}
$$

where we introduced the auxiliary functions

$$
\begin{gathered}
\left.\mathcal{I}_{n}^{\prime}(x) \equiv \frac{1}{2 \pi} \frac{\partial}{\partial \nu} \mathcal{I}_{\nu}(x)\right|_{\nu=n} \\
=\frac{i}{2 \pi} \int_{-\pi}^{\pi} \frac{\phi}{2 \pi} e^{i n \phi+x \cos \phi} \mathrm{d} \phi \\
\left.\mathcal{I}_{n}^{\prime \prime}(x) \equiv \frac{1}{(2 \pi)^{2}} \frac{\partial^{2}}{\partial \nu^{2}} \mathcal{I}_{\nu}(x)\right|_{\nu=n} \\
=-\frac{1}{2 \pi} \int_{-\pi}^{\pi}\left(\frac{\phi}{2 \pi}\right)^{2} e^{i n \phi+x \cos \phi} \mathrm{d} \phi .
\end{gathered}
$$

The typical behavior of $\chi_{t}(1, \beta, P)$ as a function of $\beta$ and $P$ is shown in Fig. 2(a). In Fig. 2(b), one may observe the precocious scaling exhibited by the ratio $\chi_{t}(1, \beta, P) /$ $\chi_{t}(1, \beta, 1)$, when we parametrize the dependence on the coupling by means of the combination $4 \pi^{2} P \chi_{t}(1, \beta, 1)$, corresponding to a physical dimensionless quantity in the continuum limit (where it takes the asymptotic value $\frac{P}{2 \beta}$ ). Precocious scaling by use of renormalized couplings was observed in a different context in Refs. $[39,40]$.

The finite volume continuum limit of the $\theta$-dependent partition function in the $U(1)$ case is

$$
Z_{\theta}\left(1, \frac{A}{2 \beta}\right)=\sum_{n} e^{-\frac{A}{4 \beta}\left(n+\frac{\theta}{2 \pi}\right)^{2}},
$$

where we dropped the $\theta$-independent multiplicative factor $I_{0}(2 \beta)^{P}$. A corresponding expression for the topological susceptibility can easily be obtained and can be written in the form

$$
\frac{\chi_{t}(1, \beta, A)}{\chi_{t}(1, \beta, 1)}=1+2 X \frac{\partial}{\partial X} \ln Z_{0}(1, X)
$$

where $X=\frac{A}{2 \beta}$. In order to compare with continuum results (see, e.g., Ref. [41]), it must be kept in mind that $g^{2}=2 e^{2}$ in the $U(1)$ case to preserve the canonical normalization of the fields (see also the note at the end of Sec. II).

In the infinite volume limit, the dominant term of the sum in Eq. (40) is the one corresponding to the minimum value of $n+\frac{\theta}{2 \pi}$, and we thus see the emergence of a multibranched structure,

$$
Z_{\theta}(1, \beta, P) \stackrel{P \rightarrow \infty}{\rightarrow}\left[\mathcal{I}_{\frac{\theta \bmod 2 \pi}{2 \pi}}(2 \beta)\right]^{P},
$$

with the partition function being nonanalytic at the odd multiples of $\pi$. This phenomenon persists also when
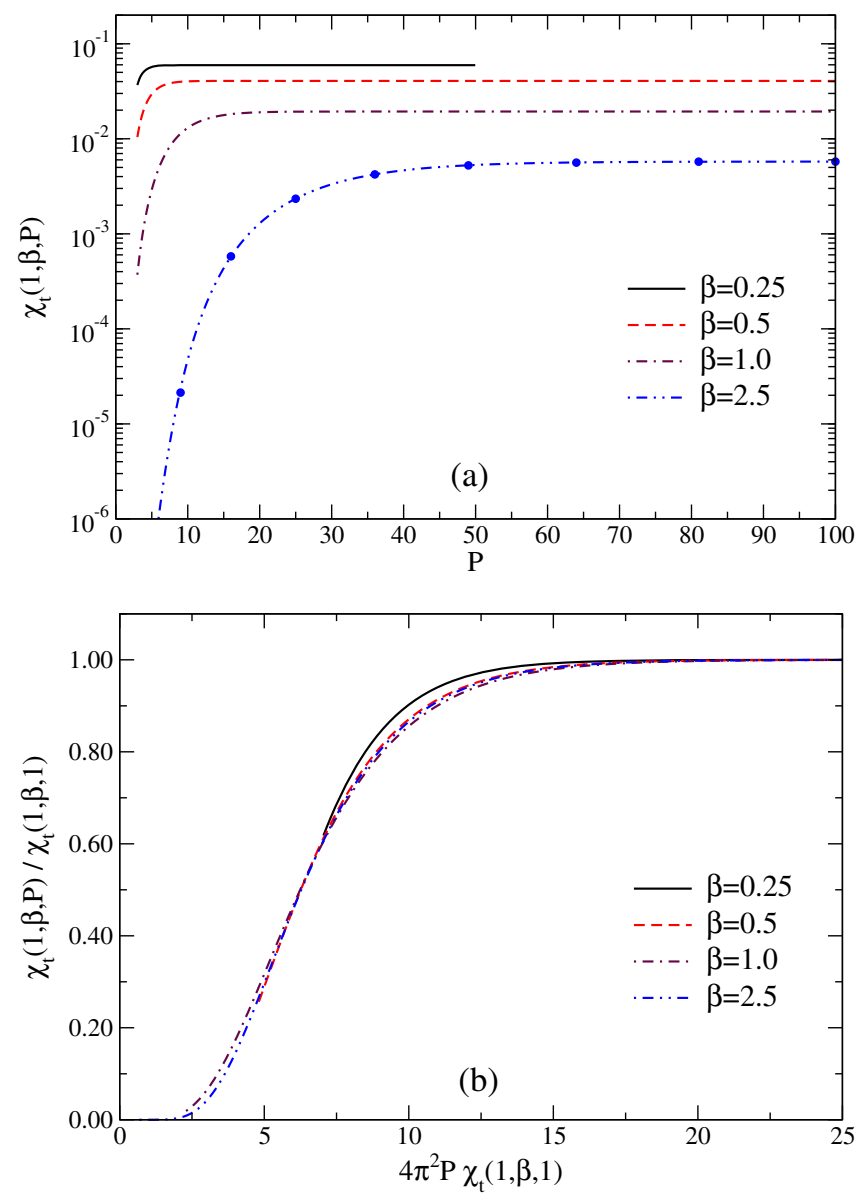

FIG. 2. (a) Behavior of the $U(1)$ topological susceptibility as a function of the coupling $\beta$ and of the dimensionless volume $P$. For comparison, points obtained by using numerical lattice simulations are also shown in the case $\beta=2.5$ (error bars are smaller than symbols). (b) Same data as in the upper panel but with quantities normalized by using $\chi_{t}(1, \beta, 1)$.

considering the infinite volume limit of the continuum version of the model discussed above. The presence of these first order transition points prevents a simple factorization of the form Eq. (10) from being applicable for generic $\theta$ values, indeed a naive application of factorization would give

$$
Z_{\theta}(1, \beta, P) \stackrel{P \rightarrow \infty}{\rightarrow}\left[\mathcal{I}_{\frac{\theta}{2 \pi}}(2 \beta)\right]^{P},
$$

which is nonperiodic in $\theta$. It is, however, important to stress that, as far as we consider $-\pi \leq \theta \leq \pi$, all the expressions obtained by using the single plaquette model correctly describe the $P \rightarrow \infty$ limit of the $P$ plaquette model. In particular, the infinite volume topological susceptibility is given by

$$
\chi_{t}(1, \beta, \infty)=-\frac{\mathcal{I}_{0}^{\prime \prime}(2 \beta)}{I_{0}(2 \beta)}
$$




\section{CONTINUUM LIMIT}

The continuum limit of two-dimensional $U(N)$ gauge theories is simply the limit $\beta \rightarrow \infty$ because the coupling $g^{2}$ is dimensionful, and therefore the above limit is the same as the limit $a^{2} \rightarrow 0$. By generalizing the arguments that lead to Eq. (13), we may obtain the following representation for the functions $\tilde{\gamma}_{r}(N, \beta, \theta)$ appearing in $Z_{\theta}(N, \beta, P)$ :

$$
\begin{aligned}
\tilde{\gamma}_{\left\{l_{j}\right\}}(N, \beta, \theta)= & \int \operatorname{det}\left[e^{i \phi_{j}\left(l_{j}+i-j\right)}\right] \\
& \times e^{i \frac{\theta}{2 \pi} \sum_{j} \phi_{j}} e^{2 N \beta \sum_{j} \cos \phi_{j}} \prod_{j} \frac{\mathrm{d} \phi_{j}}{2 \pi} .
\end{aligned}
$$

In the $\beta \rightarrow \infty$ limit, one may replace $\cos \phi_{j}$ with $1-\frac{1}{2} \phi_{j}^{2}$ and perform the resulting Gaussian integration, thus obtaining

$$
\tilde{\gamma}_{\left\{l_{j}\right\}}(N, \beta, \theta) \rightarrow A(N, \beta) \operatorname{det}\left[e^{-\frac{1}{4 N(}\left(l_{j}+i-j+\frac{\theta}{2 \pi}\right)^{2}}\right],
$$

where the common factor $A(N, \beta)$ does not depend on $\theta$.

A few straightforward manipulations allow one to represent the above result in the form

$$
\begin{aligned}
\tilde{\gamma}_{\left\{l_{j}\right\}}(N, \beta, \theta) \rightarrow & A(N, \beta) \operatorname{det}\left[e^{-\frac{1}{4 N \beta}\left(l_{j}+i-j\right)^{2}}\right] \\
& \times e^{-\frac{1}{4 N \beta}\left[\frac{\theta}{\pi} \sum_{j} l_{j}+N\left(\frac{\theta}{2 \pi}\right)^{2}\right] .}
\end{aligned}
$$

The determinant can be computed in the limit $\beta \rightarrow \infty$, obtaining the result

$$
\operatorname{det}\left[e^{-\frac{1}{4 N \beta}\left(l_{j}+i-j\right)^{2}}\right] \rightarrow B(N, \beta) d_{\left\{l_{j}\right\}} e^{-\frac{1}{4 N \beta} C_{\left\{l_{j}\right\}}},
$$

where $B(N, \beta)$ is another common factor independent of $\theta$, and it is possible to verify that the product $A(N, \beta) B(N, \beta)$ is nothing but the asymptotic form of $\tilde{\beta}_{0}(N, \beta)$ in the large $\beta$ limit; hence, it is a lattice artifact that can be ignored when analyzing the continuum properties of the model.

We recall that $C_{\left\{l_{j}\right\}}$ is the quadratic Casimir of the representation, as expected from the $\theta=0$ result (8). We report here, for the convenience of the reader, the known explicit form of $C_{\left\{l_{j}\right\}}$ and $d_{\left\{l_{j}\right\}}$ :

$$
\begin{aligned}
C_{\left\{l_{j}\right\}} & =\sum_{i=1}^{N} l_{i}\left(l_{i}-2 i+N+1\right) \\
d_{\left\{l_{j}\right\}} & =\prod_{i>j}\left(1-\frac{l_{i}-l_{j}}{i-j}\right) .
\end{aligned}
$$

The continuum limit of the partition function on a manifold with (dimensionless) area $A /(N \beta)=g^{2} V$ is therefore

$$
Z_{\theta}^{(g)}\left(N, \frac{A}{2 \beta}\right)=\sum_{\left\{l_{j}\right\}} d_{\left\{l_{j}\right\}}^{2-2 g} e^{-\frac{A}{4 N \beta}\left[C_{\left\{l_{j}\right\}}+\frac{\theta}{\pi} \sum_{j} l_{j}+\frac{N}{4 \pi^{2}} \theta^{2}\right]}
$$

and the continuum limit of the weights defined in Eq. (26) is

$w_{\left\{l_{j}\right\}}^{(g)}\left(N, \frac{A}{2 \beta}\right)=d_{\left\{l_{j}\right\}}^{2-2 g} e^{-\frac{A}{4 N \beta} C_{\left\{l_{j}\right\}}}\left[Z_{0}^{(g)}\left(N, \frac{A}{2 \beta}\right)\right]^{-1}$.

An immediate consequence of the above results is the possibility of evaluating the finite volume continuum limit of the topological susceptibility,

$\chi_{t}^{(g)}(N, \beta, A)=\frac{1}{8 \pi^{2} \beta}\left[1-\frac{A}{2 \beta} \sum_{\left\{l_{j}\right\}} w_{\left\{l_{j}\right\}}^{(g)}\left(\sum_{j} \frac{l_{j}}{N}\right)^{2}\right]$,

which in the infinite volume limit does not depend on the genus and becomes simply

$$
\chi_{t}^{(g)}(N, \beta, \infty)=\frac{1}{8 \pi^{2} \beta},
$$

for all $N$, because $w_{r}^{(g)}(N, \beta, A) \rightarrow \delta_{r, 0}$ when $A \rightarrow \infty$.

It is important to note that the continuum expression for the partition function is consistent with the previously proven periodicity in $\theta$ with period $2 \pi$ of the partition function. Let us focus on the exponents appearing in Eq. (55) and notice that they can be rewritten in the form

$$
\begin{aligned}
& C_{\left\{l_{j}\right\}}+\frac{\theta}{\pi} \sum_{j} l_{j}+\frac{N}{4 \pi^{2}} \theta^{2} \\
& =\sum_{j}\left[\left(l_{j}+\frac{\theta}{2 \pi}\right)^{2}+(N+1-2 j)\left(l_{j}+\frac{\theta}{2 \pi}\right)\right] ;
\end{aligned}
$$

also in the continuum, $\theta \rightarrow \theta+2 \pi$ is thus equivalent to $\left\{l_{j}\right\} \rightarrow\left\{l_{j}^{\prime}\right\}$, where $l_{j}^{\prime}=l_{j}+1$. Since $d_{\left\{l_{j}^{\prime}\right\}}=d_{\left\{l_{j}\right\}}$, the periodicity of the continuum partition function (55) follows as in Sec. III.

The continuum version of the $g \rightarrow \infty$ limit is simply

$$
Z_{\theta}^{(\infty)}\left(N, \frac{A}{2 \beta}\right)=\sum_{l} e^{-\frac{A}{4 \beta}\left(l+\frac{\theta}{2 \pi}\right)^{2}},
$$

and one may appreciate that it turns out to be independent of $N$ and therefore coincident with the continuum version of the $U(1)$ model. However, we notice that, contrary to naive expectations, the finite volume continuum limit will not in general coincide with its $U(1)$ value and will depend on $N$ and $g$, with the notable exception of the large $N$ limit, to be discussed in Sec. VII.

The properties of the finite volume continuum limit will be discussed in detail in a forthcoming publication. 


\section{INFINITE VOLUME LIMIT}

We assume in this section $-\pi \leq \theta \leq \pi$ (see the discussion in Sec. IV), in order to exploit the large volume factorization also at $\theta \neq 0$, obtaining for all genuses

$$
Z_{\theta}^{(g)}(N, \beta, P) \stackrel{P \rightarrow \infty}{\rightarrow} Z_{\theta}(N, \beta, 1)^{P},
$$

where

$$
\begin{aligned}
Z_{\theta}(N, \beta, 1) & \equiv \int e^{\frac{\theta}{2 \pi} \operatorname{Tr} \ln U+N \beta\left(\operatorname{Tr} U+\operatorname{Tr} U^{\dagger}\right)} \mathrm{d} U \\
& =\tilde{\gamma}_{0}(N, \beta, \theta) .
\end{aligned}
$$

Computing the infinite volume topological susceptibility thus amounts to evaluating the quantity

$$
\chi_{t}(N, \beta, 1)=-\frac{\tilde{\gamma}_{0}^{\prime \prime}(N, \beta)}{\tilde{\beta}_{0}(N, \beta)},
$$

where we exploited the fact that $\tilde{\gamma}_{0}^{\prime}(N, \beta)=0$ and the property

$$
w_{r}^{(g)}(N, \beta, P) \rightarrow \delta_{r, 0}
$$

in the limit $P \rightarrow \infty$. $\tilde{\gamma}_{0}^{\prime \prime}$ may be evaluated starting from

$$
\tilde{\gamma}_{0}^{\prime \prime}=-\int d \mu(\phi)\left(\sum_{i} \frac{\phi_{i}}{2 \pi}\right)^{2} e^{2 N \beta \sum_{i} \cos \phi_{i}}
$$

and it can be seen (using again arguments analogous to those of Refs. $[25,26,32])$ that $\tilde{\gamma}_{0}^{\prime \prime}$ may be expressed as the sum of the $N^{2}$ determinants obtained from $\operatorname{det} I_{i-j}(2 N \beta)$ by replacing one of the lines with $\mathcal{I}_{i-j}^{\prime \prime}(2 N \beta)$ and two different lines with $\mathcal{I}_{i-j}^{\prime}(2 N \beta)$. Using these expressions, it is straightforward to numerically compute $\chi_{t}(N, \beta, 1)$, and in Fig. 3, we show the results obtained for $N<10$ and

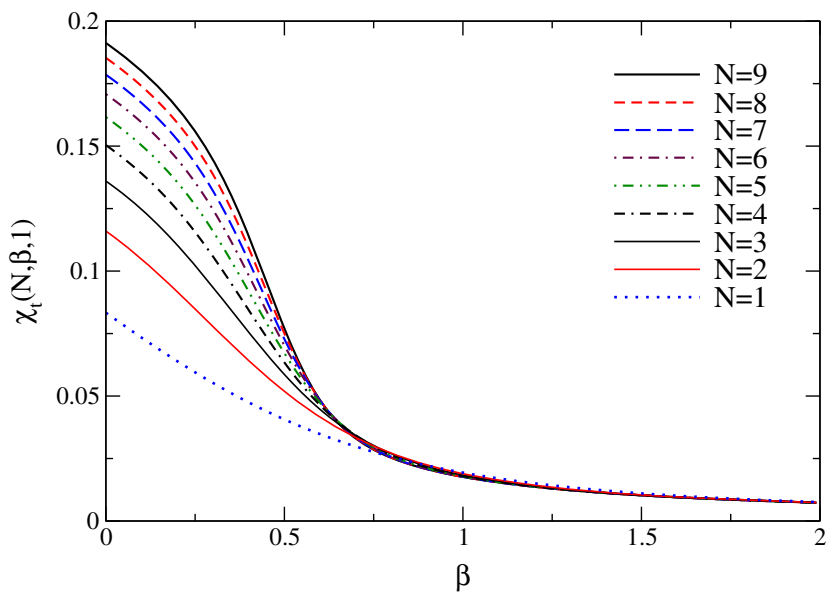

FIG. 3. Behavior of the infinite volume topological susceptibility for $U(N)$ with $N<10$ and $0 \leq \beta \leq 2$, computed using Eq. (63).
$0 \leq \beta \leq 2$; two different regimes are clearly visible in this figure, which will be discussed in depth in Sec. VII.

\section{LARGE $N$ LIMIT}

In the large $N$ limit, analytic calculations are made possible by the fact that the functional integral is dominated by the saddle-point configuration of the fields, which in turn can be found by solving the appropriate (saddle-point) equations for the eigenvalues $\phi_{i}$ of a matrix variable. In practice, one must replace the summations over the index " $i$ " with an integration in the variable $\phi$, weighted by an eigenvalue density $\rho(\phi)=\frac{1}{N} \frac{d i}{d \phi}$, normalized to $\int d \phi \rho(\phi)=1$.

Explicit eigenvalue densities have been found for the infinite volume case (equivalent to the single plaquette) [32] and for the chiral chains with $P=2,3,4$ [27,28], and the corresponding free energies have been computed. In all cases, a third order phase transition is present, and therefore one needs to know the separate expressions for the strong and weak coupling eigenvalue distributions. As we saw in the previous sections, as far as we are interested in the topological susceptibility (or in other properties related to the behavior of the free energy close to $\theta=0$ ), we can use the single plaquette model to compute values in the thermodynamic limit.

In the single plaquette model, the transition occurs at $\beta_{c}=\frac{1}{2}$, and the eigenvalue density is [32]

$$
\rho(\phi, \beta)= \begin{cases}\rho_{s}(\phi, \beta) & \text { if } \beta \leq \beta_{c},|\phi| \leq \pi \\ \rho_{w}(\phi, \beta) & \text { if } \beta>\beta_{c},|\phi| \leq \phi_{c}\end{cases}
$$

where $\phi_{c}=2 \arcsin \sqrt{1 /(2 \beta)}$ and

$$
\begin{gathered}
\rho_{s}(\phi, \beta)=\frac{1}{2 \pi}(1+2 \beta \cos \phi) \\
\rho_{w}(\phi, \beta)=\frac{2 \beta}{\pi} \cos \frac{\phi}{2}\left(\frac{1}{2 \beta}-\sin ^{2} \frac{\phi}{2}\right)^{\frac{1}{2}} .
\end{gathered}
$$

In order to extend these results to the evaluation of the large $N$ limit of the topological susceptibility at infinite volume, we must replace the saddle-point equation introduced in Ref. [32] with

$\mathrm{P} \int_{-\phi_{c}}^{+\phi_{c}} \rho\left(\phi^{\prime}, \beta\right) \cot \frac{\phi-\phi^{\prime}}{2} d \phi^{\prime}-2 \beta \sin \phi+i \frac{\hat{\theta}}{2 \pi}=0$,

where we introduced the scaling variable $\hat{\theta}=\theta / N$ in order to obtain a consistent large $N$ limit, in analogy with the procedure adopted in Refs. $[42,43]$ following the original proposal by Witten [44]. We may introduce in the saddlepoint equation the ansatz

$$
\rho(\phi, \beta)=\rho_{0}(\phi, \beta)+i \frac{\hat{\theta}}{2 \pi} \rho_{1}(\phi, \beta),
$$


where $\rho_{0}(\phi, \beta)$ is the eigenvalue density in Eq. (66) (found in Ref. [32]), while $\rho_{1}(\phi, \beta)$ must be an odd function of $\phi$ satisfying the equation

$$
\mathrm{P} \int_{-\phi_{c}}^{+\phi_{c}} \rho_{1}\left(\phi^{\prime}, \beta\right) \cot \frac{\phi-\phi^{\prime}}{2} \mathrm{~d} \phi^{\prime}+1=0 .
$$

If we denote by $\mathcal{F}(\beta, \theta)$ the free energy of the system, its $\theta$ dependent part $F(\beta, \theta) \equiv \mathcal{F}(\beta, \theta)-\mathcal{F}(\beta, 0)$ is therefore ${ }^{3}$

$$
F(\beta, \theta)=-\frac{1}{2}\left(\frac{\theta}{2 \pi}\right)^{2} \int_{-\phi_{c}}^{\phi_{c}} \rho_{1}(\phi, \beta) \phi d \phi,
$$

with the factor $1 / 2$ coming from the partial cancellation of the two terms in the free energy that are quadratic in $\theta$, i.e., the $\theta$-term and the term coming from the Haar measure. In the large $N$ limit, the above expression is finite, while all contributions of higher order in $\theta$ are depressed by powers of $1 / N$. Hence, we immediately obtain the large $N$ relationship

$$
\chi_{t}(N, \beta, 1) \rightarrow \frac{1}{4 \pi^{2}} \int_{-\phi_{c}}^{\phi_{c}} \rho_{1}(\phi, \beta) \phi d \phi .
$$

Notice that the equation defining $\rho_{1}(\phi, \beta)$ may depend on $\beta$ only through the limits of the integration domain, which in turn should not change with respect to the domain of $\rho_{0}$, because all change in $\phi_{c}$ would be depressed by a power of $1 / N$. This observation implies that special care will be needed in the strong coupling region because $\phi_{c}=\pi$ with no apparent dependence on $\beta$, but the formal solution for $\rho_{1, s}$ is

$$
\rho_{1, s}(\phi, \beta)=\frac{1}{2 \pi} \tan \frac{\phi}{2}, \quad \beta<\frac{1}{2},
$$

implying a nonintegrable singularity around $\pm \pi$. It is easy to be convinced that the resulting singular behavior may be parametrized by

$$
\chi_{t, s}(N, \beta, 1) \rightarrow \chi_{t, s}(N, 0,1)+f(\beta),
$$

where

$$
\begin{aligned}
\chi_{t, s}(N, 0,1) & =\frac{N}{12}-\frac{1}{2 \pi^{2}} \sum_{k=1}^{N} \frac{N-k}{k^{2}} \\
& \rightarrow \frac{1}{2 \pi^{2}}\left(\ln N+\gamma_{E}+1\right)+O\left(N^{-1}\right)
\end{aligned}
$$

and $f(\beta)$ is a regular function connected to the $\beta$-dependent cutoff scale, which is in turn related to the behavior of the density $\rho_{0, s}$ in the proximity of $\pm \pi$. On these grounds, since $\rho_{0, s} \sim(1-2 \beta)$ when $\phi \rightarrow \pm \pi$, we find

\footnotetext{
${ }^{3}$ This expression is clearly non- $2 \pi$-periodic in $\theta$, as a consequence of the use of the single plaquette model.
}

$$
f(\beta)=\frac{1}{2 \pi^{2}} \ln (1-2 \beta),
$$

which shows the correct $\beta \rightarrow 0$ limit and exhibits a divergence in the limit $\beta \rightarrow 1 / 2$, as required in order to match the weak coupling behavior.

In the weak coupling regime $\beta>1 / 2$, the solution of Eq. (71) is

$$
\rho_{1, w}(\phi, \beta)=\frac{1}{2 \pi} \frac{\sin (\phi / 2)}{\sqrt{\frac{1}{2 \beta}-\sin ^{2}(\phi / 2)}},
$$

the integral in Eq. (73) is convergent, and we get (using Eq. (3.842.2) of Ref. [45])

$$
\chi_{t, w}(N, \beta, 1) \rightarrow-\frac{1}{4 \pi^{2}} \ln \left(1-\frac{1}{2 \beta}\right) .
$$

This result can be easily obtained also without explicitly solving the saddle-point equation because from the definition of the topological charge we have

$$
F(\beta, 2 \pi \ell)=\ln \left\langle\operatorname{det} U_{p}^{\ell}\right\rangle
$$

and in Ref. [38] it has been proven that, at $N=\infty$ in the weak coupling phase of the single plaquette model, we have

$$
\left\langle\operatorname{det} U_{p}\right\rangle=\left(1-\frac{1}{2 \beta}\right)^{\frac{1}{2}}
$$

this was further strengthened in Ref. [46] by showing that

$$
\left\langle\operatorname{det} U_{p}^{\ell}\right\rangle=\left(1-\frac{1}{2 \beta}\right)^{\ell^{2} / 2} \text {. }
$$

Hence, we may establish the relationship, holding for all $\ell$ and $\beta>1 / 2$,

$$
F(\beta, 2 \pi \ell)=-\frac{\ell^{2}}{2} \int_{-\phi_{c}}^{\phi_{c}} \rho_{1}(\phi, \beta) \phi d \phi=\frac{\ell^{2}}{2} \ln \left(1-\frac{1}{2 \beta}\right),
$$

implying immediately

$$
\chi_{t, w}(N, \beta, 1) \rightarrow-\frac{1}{4 \pi^{2}} \ln \left(1-\frac{1}{2 \beta}\right) .
$$

This result reproduces the correct large $\beta$ behavior of the susceptibility and shows a divergence for $\beta \rightarrow 1 / 2$, needed in order to match the strong coupling behavior. Notice that, due to the singularity in $N$, this argument could not be applied to the strong coupling phase, where it is known that 

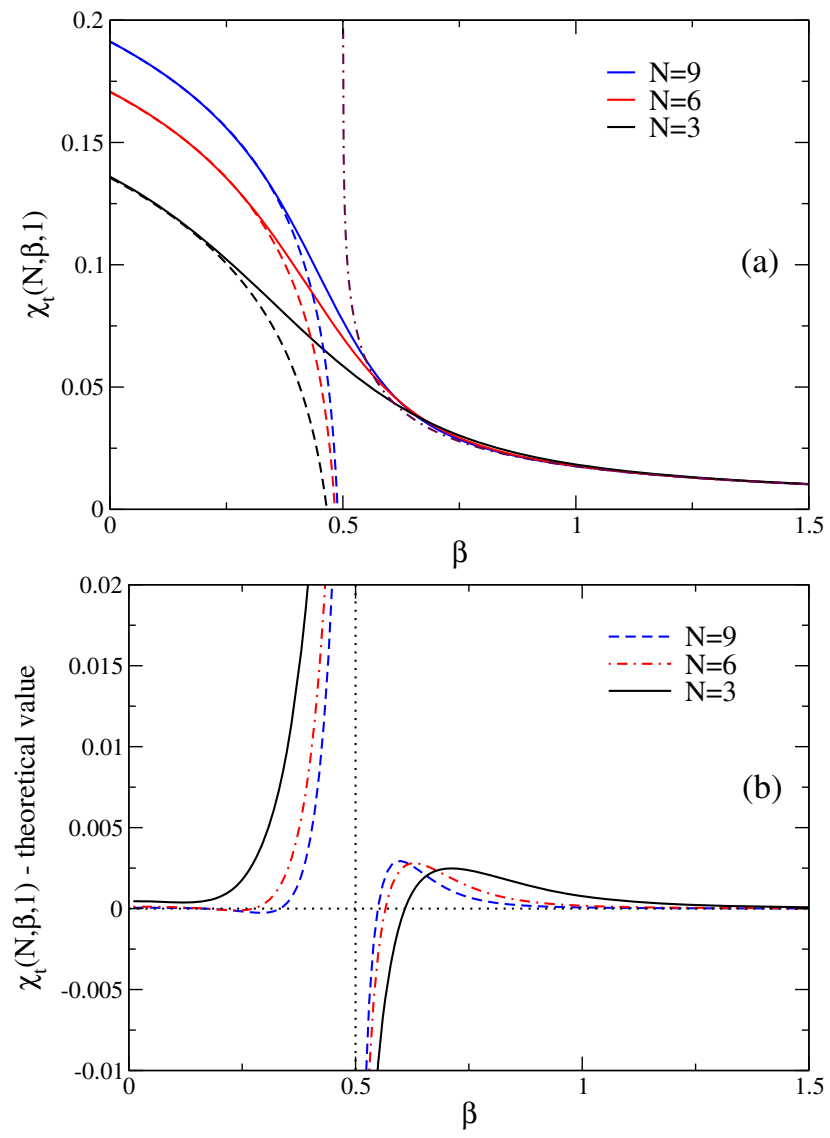

FIG. 4. (a) Comparison of numerical data obtained by using Eq. (63) (solid lines) and the leading order large $N$ theoretical predictions, which is given by Eqs. (75)-(77) for $\beta<1 / 2$ (dashed lines) and by Eq. (79) for $\beta>1 / 2$ (dotted-dashed line). (b) Deviations of numerical data from their expected asymptotic behavior.

$\ln \left\langle\operatorname{det} U_{p}\right\rangle$ is proportional to $N$ and behaves like $\ln \beta$ when $\beta \rightarrow 0[38,47,48]$.

The numerical evaluation of $\chi_{t}$, even for quite small values of $N$, shows surprisingly good agreement with the above predictions, as shown in Fig. 4.

The above results are restricted to the infinite volume version of the models, but they may be employed in the $g \rightarrow \infty$ limit in order to obtain for this case expressions holding also in the finite volume large $N$ limit, at least in the weak coupling regime. Indeed, by trivially extending Eq. (82) to include the dependence on $\theta$ and substituting the results in Eqs. (34) and (35), one easily obtains for large $N$

$Z_{\theta}^{(\infty)}(N, \beta, P) \rightarrow\left[\tilde{\beta}_{0}(N, \beta)\right]^{P} \sum_{l}\left(1-\frac{1}{2 \beta}\right)^{\frac{P}{2}\left(l+\frac{\theta}{2 \pi}\right)^{2}}$.

This expression can be rewritten in the form

$$
Z_{\theta}^{(\infty)}(N, \beta, P) \rightarrow\left[\tilde{\beta}_{0}(N, \beta)\right]^{P} Z_{\theta}(1, X)
$$

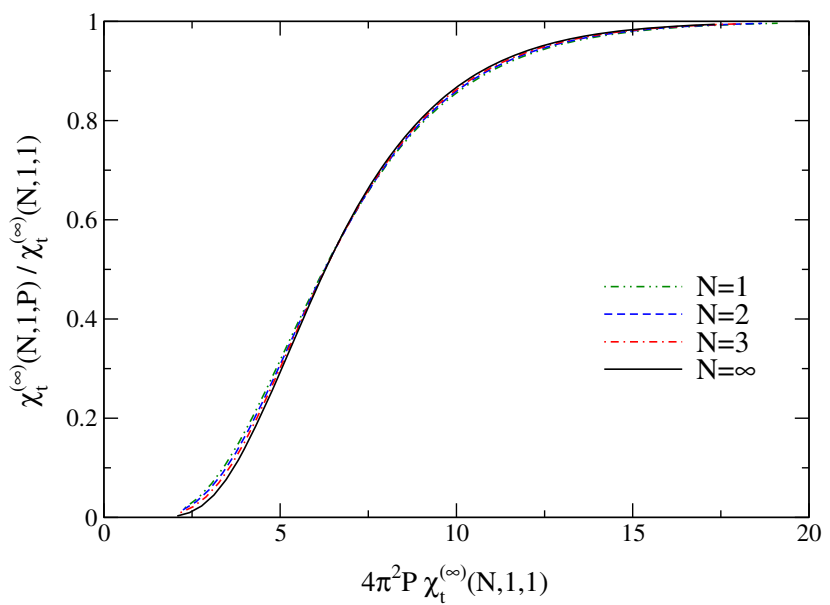

FIG. 5. Large $N$ scaling of the topological susceptibility at $g=$ $\infty$ for several values of $P$ at coupling $\beta=1$.

where $Z_{\theta}(1, X)$ is the $U(1)$ partition function of the single plaquette model (see Sec. IV) and therefore

$$
\frac{\chi_{t, w}(\infty, \beta, P)}{\chi_{t, w}(\infty, \beta, 1)}=1+2 X \frac{\partial}{\partial X} Z_{0}(1, X)
$$

where now

$$
X=4 \pi^{2} P \chi_{t, w}(\infty, \beta, 1) .
$$

Here, $\chi_{t, w}(\infty, \beta, 1)$ is the value (79) of the large $N$ limit of the topological susceptibility in the weak coupling regime, from which we may appreciate that in the continuum limit $X \rightarrow \frac{P}{2 \beta}$. It is worth noticing that very precocious large $N$ scaling is obtained when studying $\chi_{t, w}^{(\infty)}(N, \beta, P) / \chi_{t, w}^{(\infty)}(N, \beta, 1)$ as a function of the dimensionless variable $4 \pi^{2} P \chi_{t, w}(N, \beta, 1)$, which is the finite $N$ analogous of $X$; see Fig. 5. This is analogous to what was previously observed in the case of $U(1)$, shown in Fig. 2.

Another important comment concerns the dependence of the large $N$ finite volume susceptibility on $g$. It is possible to show that the same results hold true not only for $g \rightarrow \infty$ but also for all $g>1$ values because representations with $d_{r}>1$ get suppressed as $N \rightarrow \infty$ [see Eq. (26)]. On the other hand, it cannot hold in the case $g=0$, since we know that $\chi_{t}^{(0)}(N, \beta, 2)=0$ for all $N$ and, as a consequence, it vanishes also in the $N \rightarrow \infty$ limit.

By generalizing to general $g$ the arguments put forward by Douglas and Kazakov [49], one may argue that the finite area transition they found is present only in the $g=0$ case, and it would be interesting to investigate whether this transition may affect the topological susceptibility.

\section{CONCLUSIONS}

In this paper, we studied the $\theta$ dependence of twodimensional gauge theories, providing explicit expressions 
for the topological susceptibility in the most general setting, i.e., at finite volume, finite lattice spacing, and for a generic topology of the space-time manifold.

These expressions can be simplified in several different ways by restricting to more specific cases. In particular, we analyzed the thermodynamic limit at fixed ('t Hooft) coupling and the continuum limit at fixed dimensionless volume, the case of the Abelian $U(1)$ theory being particularly simple. We finally addressed the large $N$ limit of the results obtained at infinite volume, showing that the large $N$ behavior of the topological susceptibility is completely different for $\beta<1 / 2$ and for $\beta>1 / 2$. These two regions correspond to the strong and weak coupling phases of the $N=\infty$ theory, separated by the GrossWitten-Wadia transition.

From the practical point of view, our results can be useful to benchmark, in two-dimensional gauge theories, new Monte Carlo algorithms specifically targeted at improving the decorrelation of topological modes in lattice gauge theories. From the theoretical side, the most significant results obtained are probably the determination of the continuum $\theta$-dependent partition function on a manifold of arbitrary genus and the large $N$ limit (at infinite volume) of the topological susceptibility for arbitrary coupling.

A remarkable aspect of our large $N$ computation is the fact that the $\theta$ term is subleading in the action, but nevertheless, we have been able to compute the topological susceptibility at large $N$ using the saddle-point approximation method, the range of applicability of which is typically restricted to leading order computations. This is analogous to what has been done in Ref. [42] for twodimensional $C P^{N-1}$ models, but the present case is probably more surprising since the topological susceptibility does not vanish in the large $N$ limit.

Putting together the two arguments presented in Sec. VII to justify Eq. (79) we obtain a new and completely independent proof of Eq. (81), suggested in Refs. [47,48] and proven in Ref. [38], and of Eq. (82), proven in Ref. [46]. A natural question is whether the new proof can be extended to other cases that were not tractable with the previously known methods.

\section{ACKNOWLEDGMENTS}

It is a pleasure to thank M. D'Elia for useful discussions.
[1] E. Berkowitz, M. I. Buchoff, and E. Rinaldi, Phys. Rev. D 92, 034507 (2015).

[2] R. Kitano and N. Yamada, J. High Energy Phys. 10 (2015) 136.

[3] S. Borsanyi, M. Dierigl, Z. Fodor, S. D. Katz, S. W. Mages, D. Nogradi, J. Redondo, A. Ringwald, and K. K. Szabo, Phys. Lett. B 752, 175 (2016).

[4] A. Trunin, F. Burger, E.-M. Ilgenfritz, M. P. Lombardo, and M. Müller-Preussker, J. Phys. Conf. Ser. 668, 012123 (2016).

[5] C. Bonati, M. D’Elia, M. Mariti, G. Martinelli, M. Mesiti, F. Negro, F. Sanfilippo, and G. Villadoro, J. High Energy Phys. 03 (2016) 155.

[6] P. Petreczky, H. P. Schadler, and S. Sharma, Phys. Lett. B 762, 498 (2016).

[7] J. Frison, R. Kitano, H. Matsufuru, S. Mori, and N. Yamada, J. High Energy Phys. 09 (2016) 021.

[8] S. Borsanyi et al., Nature (London) 539, 69 (2016).

[9] F. Burger, E. M. Ilgenfritz, M. P. Lombardo, and A. Trunin, Phys. Rev. D 98, 094501 (2018).

[10] B. Alles, G. Boyd, M. D'Elia, A. Di Giacomo, and E. Vicari, Phys. Lett. B 389, 107 (1996).

[11] L. Del Debbio, H. Panagopoulos, and E. Vicari, J. High Energy Phys. 08 (2002) 044.

[12] L. Del Debbio, G. M. Manca, and E. Vicari, Phys. Lett. B 594, 315 (2004).

[13] S. Schaefer, R. Sommer, and F. Virotta (ALPHA Collaboration), Nucl. Phys. B845, 93 (2011).

[14] E. Vicari, Phys. Lett. B 309, 139 (1993).
[15] M. Luscher and S. Schaefer, J. High Energy Phys. 07 (2011) 036.

[16] S. Mages, B. C. Toth, S. Borsanyi, Z. Fodor, S. D. Katz, and K. K. Szabo, Phys. Rev. D 95, 094512 (2017).

[17] A. Laio, G. Martinelli, and F. Sanfilippo, J. High Energy Phys. 07 (2016) 089.

[18] W. Bietenholz, P. de Forcrand, and U. Gerber, J. High Energy Phys. 12 (2015) 070.

[19] M. Hasenbusch, Phys. Rev. D 96, 054504 (2017).

[20] C. Bonati and M. D’Elia, Phys. Rev. E 98, 013308 (2018).

[21] C. Bonati, EPJ Web Conf. 175, 01011 (2018).

[22] C. Bonati, M. D’Elia, G. Martinelli, F. Negro, F. Sanfilippo, and A. Todaro, J. High Energy Phys. 11 (2018) 170.

[23] C. Bonanno, C. Bonati, and M. D'Elia, J. High Energy Phys. 01 (2019) 003.

[24] A. M. Ferrenberg, D. P. Landau, and Y. Joanna Wong, Phys. Rev. Lett. 69, 3382 (1992).

[25] I. Bars and F. Green, Phys. Rev. D 20, 3311 (1979).

[26] J. M. Drouffe and J. B. Zuber, Phys. Rep. 102, 1 (1983).

[27] R. C. Brower, P. Rossi, and C. I. Tan, Phys. Rev. D 23, 942 (1981).

[28] R. Brower, P. Rossi, and C. I. Tan, Phys. Rev. D 23, 953 (1981).

[29] J. Kiskis, R. Narayanan, and D. Sigdel, Phys. Rev. D 89, 085031 (2014).

[30] B. E. Rusakov, Mod. Phys. Lett. A 05, 693 (1990).

[31] P. Rossi, M. Campostrini, and E. Vicari, Phys. Rep. 302, 143 (1998).

[32] D. J. Gross and E. Witten, Phys. Rev. D 21, 446 (1980). 
[33] S. R. Wadia, Phys. Lett. 93B, 403 (1980).

[34] Y. Y. Goldschmidt, J. Math. Phys. (N.Y.) 21, 1842 (1980).

[35] D. Friedan, Commun. Math. Phys. 78, 353 (1981).

[36] R. C. Brower and M. Nauenberg, Nucl. Phys. B180, 221 (1981).

[37] E. Brezin and D. J. Gross, Phys. Lett. 97B, 120 (1980).

[38] P. Rossi, Phys. Lett. 117B, 72 (1982).

[39] G. Parisi in Proceedings of the XXth International Conference on High Energy Physics, Madison (1980), edited by L. Durand and L. G. Pondrom (American Institute of Physics, New York, 1981).

[40] G. P. Lepage and P. B. Mackenzie, Phys. Rev. D 48, 2250 (1993).
[41] C. Cao, M. van Caspel, and A. R. Zhitnitsky, Phys. Rev. D 87, 105012 (2013).

[42] P. Rossi, Phys. Rev. D 94, 045013 (2016).

[43] C. Bonati, M. D'Elia, P. Rossi, and E. Vicari, Phys. Rev. D 94, 085017 (2016).

[44] E. Witten, Ann. Phys. (N.Y.) 128, 363 (1980).

[45] I. S. Gradshteyn and I. M. Ryzhik, Table of Integrals, Series and Products (Academic, New York, 2007).

[46] B. Aneva, Y. Brihaye, and P. Rossi, Phys. Lett. 133B, 215 (1983).

[47] F. Green and S. Samuel, Phys. Lett. B 103, 48 (1981).

[48] F. Green and S. Samuel, Nucl. Phys. B194, 107 (1982).

[49] M. R. Douglas and V. A. Kazakov, Phys. Lett. B 319, 219 (1993). 